\title{
Authoring a Web-enhanced interface for a new language-learning environment
}

\author{
Dominique Hémard and Steve Cushion \\ London Guildhall University, email: hemard@gu.ac.uk
}

This paper presents conceptual considerations underpinning a design process set up to develop an applicable and usable interface as well as defining parameters for a new and versatile Computer Assisted Language Learning (CALL) environment. Based on a multidisciplinary expertise combining Human Computer Interaction (HCI), Web-based Java programming, CALL authoring and language teaching expertise, it strives to generate new CALL-enhanced curriculum developments in language learning. The originality of the approach rests on its design rationale established on the strength of previously identified student requirements and authoring needs identifying inherent design weaknesses and interactive limitations of existing hypermedia CALL applications (Hemard, 1998). At the student level, the emphasis is placed on three important design decisions related to the design of the interface, student interaction and usability. Thus, particular attention is given to design considerations focusing on the need to (a) develop a readily recognizable, professionally robust and intuitive interface, (b) provide a student-controlled navigational space based on a mixed learning environment approach, and (c) promote a flexible, network-based, access mode reconciling classroom with open access exploitations. At the author level, design considerations are essentially orientated towards adaptability and flexibility with the integration of authoring facilities, requiring no specific authoring skills, to cater for and support the need for a flexible approach adaptable to specific language-learning environments. This paper elaborates on these conceptual considerations within the design process with particular emphasis on the adopted principled methodology and resulting design decisions and solutions.

\section{Introduction}

\section{The CALL legacy}

Computer-based applications in second language teaching have now been used for a protracted period of time, evolving from a deductive approach relying on grammatical progression to inductive methods and, more recently, exploratory interaction better suited 
to the constructivist mode. However, despite the initial adoption of a traditional learning environment, the first, albeit influential, generation of software design was poorly recognized, or worse, even met with scepticism by academics inasmuch as it did not seem to represent or, indeed, symbolize good teaching practices (Laurillard, 1991). As a result, original CALL programmes, such as gap-filling or substituting exercises, were often only considered appropriate as supplementary teaching material and, as such, referred to or introduced within courses as convenient adjuncts providing students with greater practical experience. Equally, students as users were never consulted on the use of CALL or, indeed, implicated beyond the designed interaction. Indeed, it was generally assumed that, since computer-based learning was a new concept, it would be, by itself, attractive and generate increased enthusiasm within the language-learning context. This situation was made even worse by a developmental process, dominated by self-taught, in-house authoring, which was too often amateurish, task-based in approach and empirical. Unfortunately, despite recent development in multimedia and hypermedia, this CALL legacy has been affecting CALL in design, practice and projected use.

\section{The hypermedia platform in CALL}

The introduction of the multimedia dimension into CALL design with its perceived interactive potential leading to hypermedia developments shed new light on the user interaction and capability of computer-based learning material. The new concepts of navigation and user-controlled access into an open environment, seen as ideal to reproduce or replicate virtual language contexts, shifted the pedagogic debate surrounding CALL to include the possibility of adding computer-based support in communicative skills and competency. However, given the evolving theoretical positions on methodological approaches to second language teaching, such a support with its inherent communicative potential also triggered questions linked to the validity of adopted learning processes and their likely outcomes. This is all the more obvious when considering the differences between language acquisition and language learning crystallized by hypermedia CALL. Moreover, in addition to the complexity of learning processes, this new interactive potential brought with it challenging design considerations focusing on user interaction, interface design, structures and strategies which required a greater range of expertise and theoretical underpinning than previously required, or thought necessary, within the design process. Noticeably, the evolution of the student-computer interaction was affecting the role played by the CALL interface and the degree of involvement and participation provided by the students themselves. Indeed, if the original approach was instructional, relying on a linear and sequential, therefore prescriptive, student interaction, the new hypermedia environment, based on student-controlled navigation, was essentially predicated upon a purposeful, independent and pro-active interaction requiring an hitherto lacking motivation and application on the part of students. Furthermore, such a student-orientated interaction coupled with the self-contained nature of this new interactive language environment and the increasing use of commercially produced packages made the integration of CALL more difficult due to poor complementarity with existing language teaching material and an overall lack of confidence displayed by the teaching staff.

To attempt to reverse such a trend, this paper purposely focuses on a theoretical approach to design, highlighted by design considerations behind a new Web-enhanced CALL 
environment supporting French, German and Spanish within the Department of Language Studies at London Guildhall University. This CALL interface was initiated on the basis of previously identified student requirements and authoring needs, which in turn, informed its design, the student interaction and its usability as well as authoring facilities.

\section{Student requirements}

Student requirements were obtained over a protracted data-gathering exercise focusing on the identification of relevant and reliable representations expressed by students on the basis of their interaction with CALL software during user walkthroughs (Bainbridge, 1990; Hémard, 1998). These student requirements were gathered by means of short meetings or verbal exchanges with the students concerned, often taking place immediately after the scheduled user walkthroughs, as well as discussions in an attempt to maximize the impact of, and impressions on the interface interacted with. These student requirements aimed at providing a valuable design support reflecting the widest possible range of perceived features in a hypermedia CALL environment on the basis of the students' experience. In this respect, they generated greater understanding and a more precise representation of student needs and abilities, helping, in the process, to contribute to the provision of student-centred data supporting the language-learning environment design process.

\section{Interface design issues}

\section{Screen display: consistency, clarity and meaningfulness}

Overall, students expressed the need for a good, professionally designed screen display, which would be consistent, clear and uncluttered, stable and reliable, which would only display relevant and useful features and whose icons, symbols and graphical representations had to be compatible and standardized. Multimedia extensions needed to be equally displayed and purposeful. The designed environment and its material had to be attractive and identifiable in order to make it easily understandable and to facilitate the learning process.

On the strength of student requirements, design considerations initially centred on the choice of environment and interface. In order to reduce cognitive overheads related to the user interface, it was agreed to adopt the most readily recognized and interacted-with Windows-based environment in the form of a Web browser. Advantages in design terms were numerous and far outweighed perceived limitations. Firstly, the screen display adopted known and agreed design standards and protocols providing easily understandable and, therefore, learnable conventions. Secondly, the Web was widely available as well as being attractive and increasingly popular amongst students. Finally, its interface was felt to be particularly intuitive, possibly as a result of a higher level of interactive exposure than with other environments, but also because it was still, bar the recent flurry of animated objects, clear, unambiguous and reasonably robust. Conversely, the adoption of a Web browser also meant that design trade-offs had to be made. By far the most crucial one was linked to the limited multimedia interaction currently available on the Net. While being confident that this was a temporary technological problem, the fact remained that when the decision was made, the Web browser was not perceived as a fullyfledged multimedia platform. If multimedia interactivity was largely traded off in the 
initial stage for increased intuitiveness and learnability, so too were personalized design characteristics. The screen display was to adopt the default background, with its accustomed display of links, commands and features, and colours were to be used parsimoniously. Finally, as an interesting offshoot of the multimedia design trade-off, it became clear during the evaluation phase that students were far more ready and willing to interact with textual material in a Web-based presentation than they had been previously in hypermedia or multimedia environments. Whilst a multimedia dimension was added at a later stage, with sound extensions, and welcomed by students, it clearly showed that students were still conditioned by the hypertext-based interactivity provided by the Web.

\section{Student interaction}

\section{Mixed learning environment}

Overwhelmingly, the students required full control over their interaction and wanted to be allowed unrestricted movement. However, the point was made that interactive aims and objectives had to be clearly stated if the student interaction was going to be purposeful. Furthermore, the interface had to provide clear and appropriate interactive support towards a self-sufficient interaction.

In many respects, the mixed learning environment, combining both student-controlled navigational commands and instructional elements, represented the most appropriate and versatile construct to be supported by a Web-browser. Capitalizing on the interactive potential of the Web, the computer could fulfil the function of a learning tool (Levy, 1998) providing a non-directive or self-directed resource whose interface, in a wider physical sense, could, similarly, accommodate the intervention or collaboration of the teacher. Furthermore, the Web browser could also support student initiatives and student-led activities and enhance authentic learning activities without the need to evaluate progress or performance. But more importantly, a mixed learning environment approach was seen as providing access to both discrete, language-based instructional activities as well as links to relevant content-based resources, thus bridging the gap between the language structure and its content. In this respect, students could benefit from curriculum-based, directed instructions providing language-learning practice and objectives as well as relevant learning resources in an interactive format. Thus, it was hoped that navigational and interactive controls, previously criticized by students for generating a negative, counterproductive interaction, as well as interactive language exercises, equally criticized for being too coercive and limited, could, together better counteract the flaws and inadequacies of the computer within the language-learning process.

\section{Structural model}

In the mixed environment mode, a root and branch or network approach with nuclei of accessible discrete composites is a flexible and appealing structure within a hypermedia CALL environment. Branching routes can conveniently accommodate specific contentbased units interconnected by associative links (Grabinger and Dunlap, 1996) with discrete sub-networks attached for greater exploration, interactive participation or specific taskbased activities. Levy (1999) likens this learning environment to a 'holistic-discrete approach', highlighting its adaptability to and appropriateness with Web technologies and access. Moreover, Jacobson (1994) highlights the interrelated and web-like nature of knowledge which needs fixed as well as variable links to connect and assemble thematic 
approaches and concepts with real applications. Therefore, its structural model, or structured learning interaction (Boyle, 1997; Kommers et al., 1996), was conceived on the basis of a student-controlled navigational space with embedded associations in the form of hypertext links, albeit supporting directional, interactive branches with sequential links, themselves accommodating more appropriate deductive and inductive learning modes.

At the design level, the clear advantage of such an approach was strategic as well as technological. Firstly, it became possible to focus on the broad structure and overall goals of the environment whilst developing isolated interactive nuclei, in the form of interactive language exercises built within Java applets, separately and therefore more manageably. At a more pragmatic and technological level, expertise and capabilities could be better spread out and adapted to a wider range of different task-based sub-components. Likewise, this structure could be used for an inductive approach wherein language tasks, requiring a more systematic and sequential approach, could be branched, and exercises and activities bunched and attached, creating controlled loops within a more exploratory environment. In this respect, task-based activities could be organized hierarchically or freely accessed and controlled by students. Finally, such a structural model, encompassing mixed learning strategies and activities as well as a variety of different and discrete interactive environments, was seen as being easily identifiable and applicable to students, who were themselves familiar with a modular programme of studies and a multi-site university. Indeed, this hypercontext (Barrett, 1994) comprises classroom teaching, but also, increasingly, self-access networked language resources designed to promote active learning, deeper contextual understanding and increased adaptability. Thus, this structural model was designed to support a wide range of student interaction linked to a different exploitation of relevant textual material, be it exploratory or directional, to focus on, for instance, listening, gap-fill, vocabulary-building and grammar exercises.

\section{Usability}

As a high-level design consideration, usability went beyond usability features of the designed application to include wider aspects related to access, assimilation and integration. Given the prevalent negative attitudes observed amongst a disenfranchised student population, it was felt that a particularly important objective of the new environment was to reintroduce and reinstate both the CALL concept and practice within the students' learning experience with a view to meeting two important requirements: specificity and applicability (Carroll, 1991).

The majority of student requirements focused on the perceived inadequacies of the functionality, limiting the usability of applications such as the complexity and prescriptive nature of tasks, warnings and locking mechanisms. However, some requirements were particularly pertinent to a higher level usability. For instance, the point was made that multimedia was not by its very nature attractive, and that, therefore, it had to be made so by design. Similarly, a recurrent concern was raised that students' own motivation needed to be maintained throughout the interaction. Overwhelmingly, students indicated that they could not see the point of using CALL applications within their own language context and felt that introductory suggestions of language-learning approaches with their recommended access modes ought to be provided to instil a greater sense of purpose and 
raison d'être into CALL. Finally, it was thought that clearer links between applications and language teaching programmes would generate a more fruitful interaction and make the design more adaptable to the different levels of student needs and language expertise. Of interest in this particular context was the fact that the students' main referential criteria were noticeably drawn from their own and often negative interaction in language laboratory conditions.

As a result, it was agreed that the best way to meet requirements such as specificity and applicability to generate a purposeful interaction was by a process of assimilation. Indeed, assimilation was to become an important objective of the project and influenced the design process at two distinct conceptual levels. Firstly, at the developmental level, since it was decided to conceive this new CALL project as part of a curriculum design process. For once, CALL was not a late addition or an after-thought, but an important and complementary element entering the programme of study. In turn, this approach supported the well-mastered drill and practice mode whilst creating new links to related textual and multimedia Web resources. Similarly, by intrinsically linking CALL and curriculum developments, members of the teaching staff, themselves potential authors, became involved in the design at an earlier stage in the process, thus facilitating a crucial exchange of ideas and objectives. By the same token; students, as end-users but also interested parties, were also encouraged to participate in the formative phase of the design by giving the design team feedback on early prototypes of interactive exercises and mixed learning interfaces, which were provided for this purpose on CD-ROMs. Therefore, staff and students were seen as partners or collaborators for this project and this protracted involvement both in the formative and in the summative stages of the process proved to be very beneficial to the overall usability of the designed interface. Secondly, at the interface level, assimilation was linked to the ability to explore the CALL environment meaningfully, allowing the learner to anticipate, recognize, identify and acquire the relevant information. Therefore, it was related to the usability factor of the application as well as cognitive overheads it generated when interacting with it both at the operational level, with language exercises, and at the informational level, when used as language resource. Thus, although students were given full interactive control, the clear strategy and sheer variety of designed interactions meant that motivation would be sustained and students more naturally inclined to be pro-active. Ultimately, it was hoped that, by increasing CALL's availability and integration into the students' experience, it would be possible to promote a flexible, network-based, access mode encompassing and reconciling classroom access and use with self- and open-access exploitations.

\section{Authoring}

In parallel, the main findings from two surveys, an international survey carried out in 1991 (Levy, 1997) and a London-based survey carried out in 1995 (Hémard, 1995), were used to sketch out an author profile and identify authoring needs to be supported by high-level design considerations. Initially, all respondents, including those without authoring experience, indicated that CALL was suitable and could improve the language-learning experience if properly integrated into the overall learning environment. Therefore, authoring applied to the provision of grammar support mechanisms as well as a more constructivist multimedia interaction. 
Of interest, the lack of general design expertise was felt to be detrimental and translated itself into poor design considerations, which made little or no mention of design potential in terms of expected student interaction, student requirements and the desired match between the task and the action domains. Similarly, Levy's survey focused on the way authors conceptualized CALL in terms of strategies, roles, modes and scope (Levy, 1997). Not unlike the previous London-based results (Hémard, 1995), respondents were perceived as being notably 'eclectic' when selecting their preferred teaching and learning approaches, showing a preference for communicative approaches whilst still supporting, although to a lesser extent, more formal, deductive methods. A similar range of responses was elicited on authors' perception of the role the computer should play. Whilst split on the question, the majority of respondents considered that the computer should play a non-directive role and saw it fundamentally as a tool. Not surprisingly, directive roles such as manager of tasks, expert system or surrogate teacher were the least favoured. Last but not least, respondents did not seem to differentiate between roles and contexts of use making the computer, regardless of its role, suitable in both the self-access mode and the classroom.

Questions related to the design process showed that the majority of respondents had difficulty in the pre-design, conceptualization phase favouring a lower-level, task-based, problem-solving approach instead of a higher- level design process. Such widely differing views also highlighted the wide range of authors involved in CALL design, from the language teacher with no prior design expertise to authors conducting research and reviews, or developers. This last point was, indeed, corroborated by the general view, expressed by respondents, that the role of the teacher as author was to be involved in CALL materials writing. Not surprisingly, therefore, the majority of responses showed the overwhelming domination of textuality in CALL indicating that the computer was still best at enhancing reading, writing and text reconstructions with listening coming first followed by interactive video, interactive audio, simulation and finally speaking. Therefore, if CALL was considered appropriate, its multimedia applicability, in terms of hardware delivery, language aspects and learning strategies, was not easily realizable. Above all, the authoring dimension in CALL was focusing on another user group, formed by language teachers, who, on the whole, were driven by the overriding need to adapt and exploit CALL with little or no design expertise and technical support.

On the strength of these characteristics, shared by many authors or would-be authors in foreign languages, high-level design considerations concentrated on specific design objectives. Firstly, given that the usability of authoring interfaces had hardly been addressed previously (Nielsen, 1995), authoring became an important concept to consider within the environment to be designed for two essential reasons. On the one hand, the creative language input needed to be authored by all relevant teachers regardless of their ICT expertise and, on the other, teaching staff had to be involved in the design process in some way if they were to be expected to integrate and use the finished interface in their teaching. Thus, an authoring tool extension had to be designed to provide content material but also built-in flexibility and, above all, ownership. However, in order to avoid reinventing the authoring wheel with its known technological pitfalls, and on the strength of existing evidence, it was decided that the only way to win over recalcitrant language teachers was to identify and, above all, separate both authoring input and function. On such a premise, the specificity of the authoring tool was to enable teachers to author in 
their own language material in their known word-processing environment, delivering data which were then to be deciphered and translated into programmed interactivity by the machine-driven tool. Therefore, by dint of separating input from output, a simple, but versatile and powerful, authoring tool, requiring no specific authoring skills, could cater for and support the realistic needs of all language teachers, who, at long last, would feel involved and, by extension, motivated into exploiting CALL. To date, this device developed in Java provides this built-in intuitive authoring approach to design gap-filling and substituting exercises, interactive tables of verbs, listening exercises, multiple choices, word identification, text jumbling and a glossary writer mode.

\section{Conclusion .}

The advent of the Internet providing increased access, recognition and realizable interactive potential, coupled with the attraction generated by cutting-edge technology, can significantly benefit CALL if properly harnessed. It is to be hoped that, by having focused specifically on the theoretical basis supporting the design process and highlighted projected usability both at the level of students and language-teaching staff, this paper will be instrumental in helping CALL to shed its tarnished reputation and enter a new developmental phase.

\section{References}

Bainbridge, L. (1990), 'Verbal protocol analysis', in J. R. Wilson and E. N. Corlett (eds.), Evaluation of Human Work: A Practical Ergonomics Methodology, London: Taylor and Francis, 161-79.

Barrett, E. (ed.) (1994), Sociomedia: Multimedia, Hypermedia, and the Social Construction of Knowledge, MIT Press.

Boyle, T. (1997), Design for Multimedia Learning. London: Prentice Hall.

Carroll, J. (1991), 'The Kittle House Manifesto', in J. Carroll (ed.), Designing Interaction: Psychology at the Human-Computer Interface, Cambridge University Press, 1-16.

Grabinger, S. and Dunlap, J. (1996), 'Links', in P. A. M. Kommers, S. Grabinger and J. C. Dunlap (eds.), Hypermedia Learning Environments, Instructional Design and Integration, Mahwah, New Jersey: Lawrence Erlbaum Associates.

Hémard, D. P. (1995), 'Design principles and guidelines for authoring hypermedia language learning applications', Design Support Project, MSc (User Interface Design), London Guildhall University, September 1995.

Hémard, D. P. (1997), 'Design principles and guidelines for authoring hypermedia language learning applications', System, 25 (1), 9-27.

Hémard, D. P. (1998), 'Knowledge representations in hypermedia CALL authoring: conception and evaluation', Computer Assisted Language Learning, 11 (3), 247-64.

Jacobson, M. (1994), 'Issues in hypertext and hypermedia research: toward a framework for linking theory-to-design', Journal of Educational Multimedia and Hypermedia, 3 (2), 141-54. 
Kommers, P. A. M., Grabinger, S. and Dunlap, J. C. (eds.) (1996), Hypermedia Learning Environments, Instructional Design and Integration, Mahwah, New Jersey: Lawrence Erlbaum Associates.

Laurillard, D. (1991), 'Principles for computer-based software design for language learning', Computer Assisted Language Learning, 4 (3), 141-52.

Levy, M. (1997), Computer-Assisted Language Learning - Context and Conceptualization, Oxford: Clarendon Press.

Levy, M. (1999), 'Design processes in CALL: integrating theory, research and evaluation', in K. Cameron (ed.), CALL: Media, Design and Applications, Swets \& Zeitlinger.

Nielsen, J. (1995), Multimedia and Hypertext: The Internet and Beyond, Boston, San Diego, NY, London, Sydney, Tokyo, Toronto: AP Professional. 